\title{
A methodology for characterizing gun barrel flexure due to vehicle motion
}

\author{
Mark Bundy ${ }^{\mathrm{a}}$, James Newill ${ }^{\mathrm{a}}$, Vince Marcopoli ${ }^{\mathrm{b}}$, \\ Michael $\mathrm{Ng}^{\mathrm{b}}$ and Charles Wells ${ }^{\mathrm{b}}$ \\ ${ }^{a}$ Army Research Laboratory, Aberdeen Proving \\ Ground, MD, USA \\ ${ }^{\mathrm{b}}$ General Dynamics Land Systems, Sterling Heights, \\ MI, USA
}

\begin{abstract}
Barrel centerline curvature is known to influence the location of projectile shot impacts. Superimposed on the unique manufactured barrel centerline is the flexed barrel shape that can occur prior to firing while the vehicle is on the move. In order to understand and quantify the effects of barrel flexure on gun accuracy, it is necessary to determine what combination of fundamental mode shapes is most likely to occur. A method to accomplish this task is described in this paper. The method is demonstrated by enumerating the 10 most likely flexed barrel shapes that were found to occur in a tank-mounted gun barrel while it traversed a bump course.
\end{abstract}

\section{Introduction}

Barrel centerline curvature is known to influence the location of projectile shot impacts. Superimposed on the unique manufactured barrel centerline is the flexed barrel shape that can occur prior to firing while the vehicle is on the move. In order to understand and quantify the effects of barrel flexure on gun accuracy, it is necessary to determine what combination of fundamental mode shapes is most likely to occur. A method to accomplish this task is described in this paper.

\section{Describing barrel motion}

In order to maintain target tracking while on the move, the gun barrel of a main battle tank is subjected to translational and rotational motion, as illustrated in Fig. 1 . In brief, the barrel is rotated by the angle, $\theta$, that is required to keep the gunner's line of sight pointed at the target while traversing uneven terrain. Rotation is produced by the action of a hydraulic actuator located on the breech side of the trunnion axis, as depicted in Fig. 2.

In addition to the rigid-body motion, flexural modes of motion will be excited by vehicle motion. Thus, the dynamic state of the prefiring barrel can be described by the following:

$$
y(x, t)=\overbrace{y_{t}(t)+\theta(t)\left[x-x_{t}\right]}^{\text {rigid body }}+\underbrace{Y(x, t)}_{\text {flexing mode }}
$$

where $y$ gives the vertical component of the lateral barrel displacement (relative to the static barrel centerline) at time $t$ in an earth-fixed coordinate frame; $x$ specifies the axial barrel coordinate relative to the breech face ( $x_{t}$ is the location of the trunnion); $y_{t}$ gives the vertical displacement of the trunnion axis; $\theta$ specifies the rotation of the barrel about the trunnion axis; and $Y$ gives the vertical component of the lateral barrel displacement (relative to the static barrel centerline) in a coordinate frame that is rotating and translating with the barrel. The flexing mode component, $Y$, can be further broken down into

$$
\begin{aligned}
Y(x, t)= & q_{1}(t) Y_{1}(x)+q_{2}(t) Y_{2}(x) \\
& +\ldots+q_{n}(t) Y_{n}(x),
\end{aligned}
$$

where $Y_{i}(i=1,2, \ldots, n)$ represent the first $n$ barrel flexing mode shapes (normalized to unity), and $q_{i}$ give the mode shape amplitudes. It is assumed in Eq. (2), and later shown to be the case, that no more than the first three mode shapes are needed to account for barrel flexing from tank motion.

It is not within the scope of the present study to quantify the effects of rigid-body or flexing mode motion on the projectile's flight path, though such a correlation is reported to exist [1-3]. It is, however, of interest to determine the dominant barrel flexing modes and, moreover, establish a methodology to ascertain the mode shape combinations that are most likely to occur prior to firing on the move. 


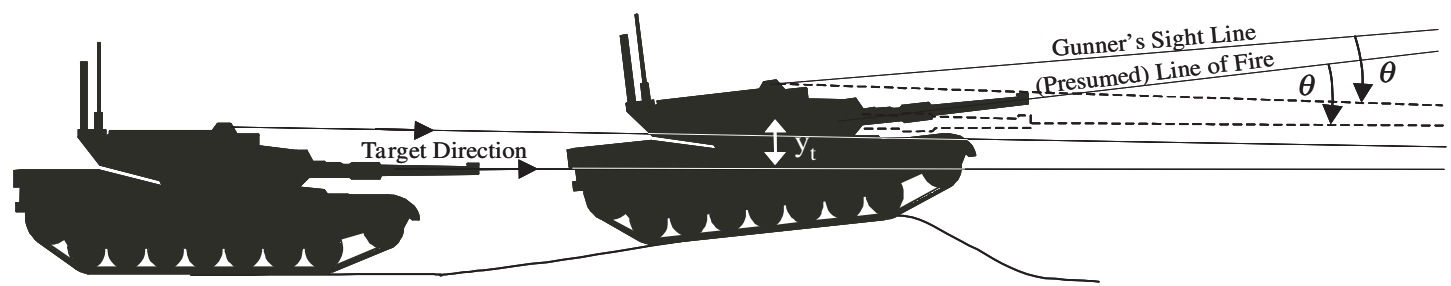

Fig. 1. Caricature of rigid-body motion of the sight and barrel while target tracking as the tank traverses a bump.

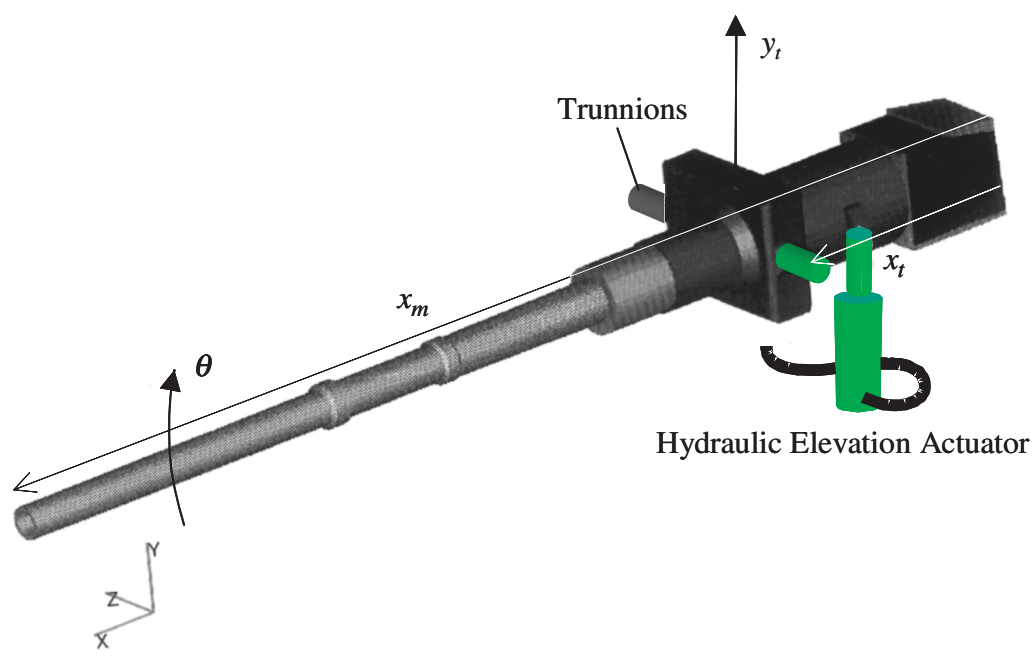

Fig. 2. Rigid-body motion of the barrel due to terrain and actuator forces.

\section{Barrel flexing modes and tube shapes}

Modeled as a system of point masses connected by spring-like forces, the fundamental barrel flexing modes can be determined by solving the appropriate mass-stiffness matrix. The first three mode shapes for the M256 120-mm barrel are shown in Fig. 3; all shapes are normalized to unity. Note that all three mode shapes pass through 0 at the location of the trunnions $(\sim 53 \mathrm{in}$, $1.35 \mathrm{~m}$ ) and actuator ( $~ 35 \mathrm{in}, 0.89 \mathrm{~m}$ ), where the point masses are considered to be pinned. As inferred in the previous Section, rigid-body motion accounts for the displacement as well as rotation of the line passing through the actuator and trunnion points (Eq. (1)) relative to which the mode shapes of Fig. 3 are referenced.

\section{Flexure due to vehicle motion}

The shape of the flexed barrel at any given time is determined by the mode shape amplitudes, $q_{i}$, according to Eq. (2). Figure 4 shows a typical time sample of the mode shape amplitudes for the M256 barrel mounted on the M1A2 tank, traversing the bump course referred to as RRC-9 at Aberdeen Proving Ground, MD. As indicated, the first mode is the dominant one. While traversing RRC-9, the ratio of amplitudes $q_{1}: q_{2}: q_{3}$ was found to be on the order of 25:5:1. This being the case, it is possible to adequately characterize/model flexing of the M256 barrel over this course by summing over just the first two mode shapes in Eq. (2).

Figure 5 illustrates how the ratio of $q_{1} / q_{2}$ can be discretized by counting the number of occasions that a given ratio (within some tolerance band) occurs over a given time span. Figure 6(a) shows how the distribution of ratios $q_{1} / q_{2}$ varies, over the range from -50 to +50 in increments of 1 , while an M1A2 tank traversed RRC-9 at $15 \mathrm{mph}$ (taking an elapsed time of $20 \mathrm{~s}$ and generating 9,000 data samples). (This type of accounting/plotting will fluctuate somewhat with the points selected and the width of the increments, but in general, trends will be independent of these factors.) For instance, it can be seen from Fig. 6(a) that values of $q_{1} / q_{2}$ between -1.5 and +0.5 occurred most often, 523 times out of the 9,000 time increments sampled (this same peak location occurred when the sampling was refined tenfold). Figure 6(b) shows the equivalent 


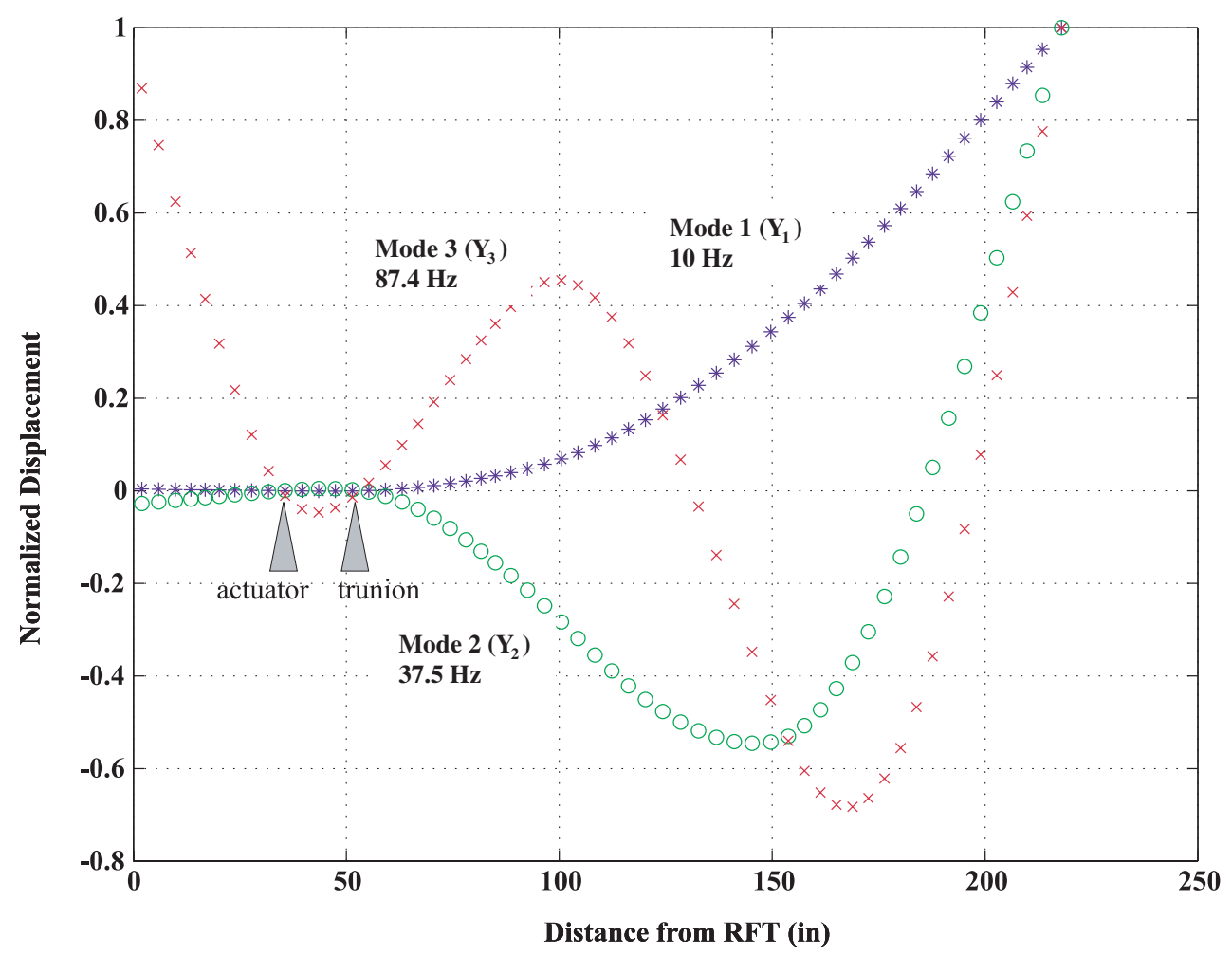

Fig. 3. First three fundamental barrel flexing mode shapes (normalized to unity) for the M256 120-mm barrel.

result plotted in the nondimensional format of relative frequency of occurrence.

The 10 most likely ratios of $q_{1} / q_{2}$ are displayed in Table 1, where it can be seen that they are nearly equal in likelihood of occurrence, ranging from a low of $3.4 \%$ (case 10) to a high of 5.8\% (case 1). However, there is a very gradual trend that shows higher values of $q_{1} / q_{2}$ are less likely to occur; this observation is shown more dramatically in the plots of Fig. 6.

Figure 7 shows the barrel flexure profiles corresponding to each of the top 10 ratios listed in Table 1 . The most undulating shapes are those with the highest proportion of second mode motion (e.g., cases 1-5). For the most part, the higher amplitude shapes are those dominated by first mode motion (e.g., cases 6-10).

The top 10 tube shapes in Table 1 and Fig. 7 were obtained by searching the database of Fig. 6(b) for any case where the frequency of occurrence exceeded $3 \%$. When this search window is broadened to include any case where the probability was greater than $0.5 \%, 37$ ratios are found that meet this criterion. These 37 cases, plotted in Fig. 8, represent the flexed barrel profile for a combined $82.5 \%$ of the bump course transit time. The spread in peak amplitudes across this group is roughly 0.030 in $(0.76 \mathrm{~mm})$. Also shown in Fig. 8, for comparison, is the average manufactured centerline shape for the M256 barrel [4]. For the most part, the spread in manufactured barrel centerlines, as registered by their (peak) muzzle displacements, spans about three times this spread in muzzle displacements.

\section{Summary and conclusions}

This report documents a methodology to assess the effects of tank motion on the static barrel centerline shape of a rigidly rotating and translating gun barrel. It has been shown, for example, that for an M256 barrel in an M1A2 tank traversing the RRC-9 bump course at $15 \mathrm{mph}$, the resulting barrel flexure could be adequately captured by a linear combination of just the first two mode shapes (higher order mode shapes were not a significant factor). For roughly a third (34\%) of the bump course transit time, the ratio of the first to second mode amplitudes was $\leqslant 3$. Moreover, for this mixed-mode group, the median mode shape amplitudes were relatively small. The remaining two-thirds of the time, the barrel was essentially exhibiting first-mode 


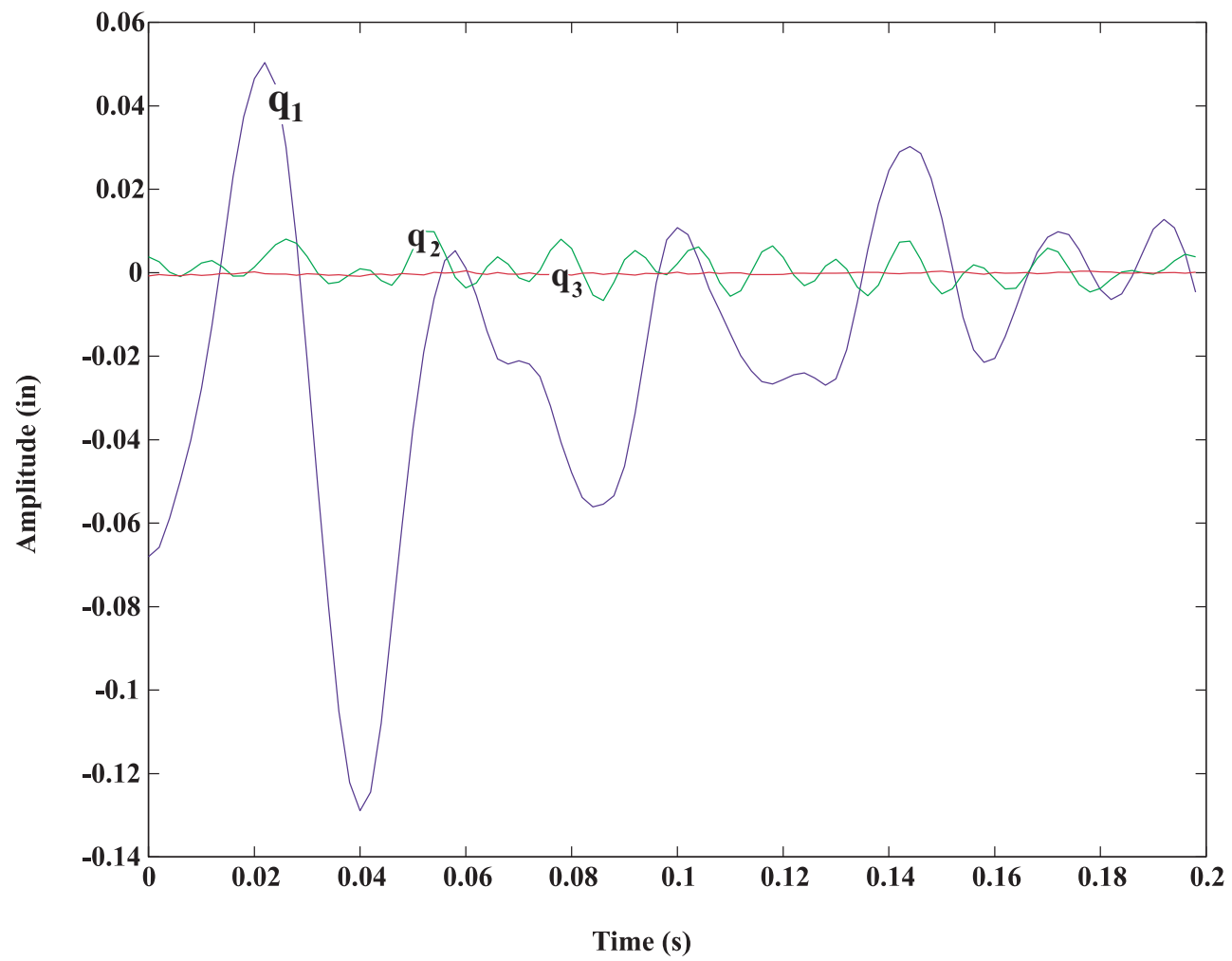

Fig. 4. Typical mode shape amplitudes while vehicle is in motion.

Table 1

Ten most likely ratios of $q_{1} / q_{2}$

\begin{tabular}{ccccc}
\hline $\begin{array}{c}\text { Probability } \\
\text { order }\end{array}$ & $\begin{array}{c}\text { Ratio of } q_{1} / q_{2} \\
( \pm 0.5)\end{array}$ & $\begin{array}{c}\text { Frequency of } q_{1} / q_{2} \\
\text { occurring }(\%)\end{array}$ & $\begin{array}{c}\text { Median value of } q_{1} \\
\left.\text { (inches } \times 10^{-3}\right)\end{array}$ & $\begin{array}{c}\text { Median value of } q_{2} \\
\left(\text { inches } \times 10^{-3}\right)\end{array}$ \\
\hline 1 & -1.0 & 5.8 & -1.5 & +1.7 \\
2 & -2.0 & 5.4 & -5.6 & +3.0 \\
3 & -3.0 & 5.2 & -5.8 & +2.0 \\
4 & +0.0 & 5.2 & -0.0 & -1.0 \\
5 & +1.0 & 4.5 & -0.9 & -1.1 \\
6 & -4.0 & 4.2 & -6.9 & +1.7 \\
7 & +2.0 & 4.0 & -4.0 & -2.1 \\
8 & +3.0 & 3.8 & -5.6 & -1.9 \\
9 & -5.0 & 3.7 & -8.0 & +1.6 \\
10 & -6.0 & 3.4 & -7.6 & +1.2 \\
\hline
\end{tabular}

motion, albeit with relatively large amplitude, such that the spread in peak muzzle displacement over more than $80 \%$ of the bump course transit time was equivalent to roughly a third of the spread in statically measured muzzle displacements for barrels coming off the current production line.

\section{References}

[1] M. Bundy and B. Patton, Variation in Shot Impacts Due to Controlled Bending of a Gun Tube, ARL-TR-1190, US Army Re- search Laboratory, Aberdeen Proving Ground, MD, September 1996.

[2] J.M. Garner, M.L. Bundy, D.W. Webb and B.J. Patton, Variation in Muzzle Pointing Angle and Shot Impact of the M242 Chain Gun, ARL-TR-785, US Army Research Laboratory, Aberdeen Proving Ground, MD, July 1995.

[3] S. Wilkerson, Possible Effects of Gun Tube Straightness on Dispersion, ARL-TR-767, US Army Research Laboratory, Aberdeen Proving Ground, MD, June 1995.

[4] S. Wilkerson, Private communications on the average measured centerline profile for the M256 120-mm barrel, US Army Research Laboratory, Aberdeen Proving Ground, MD, October 1998. 


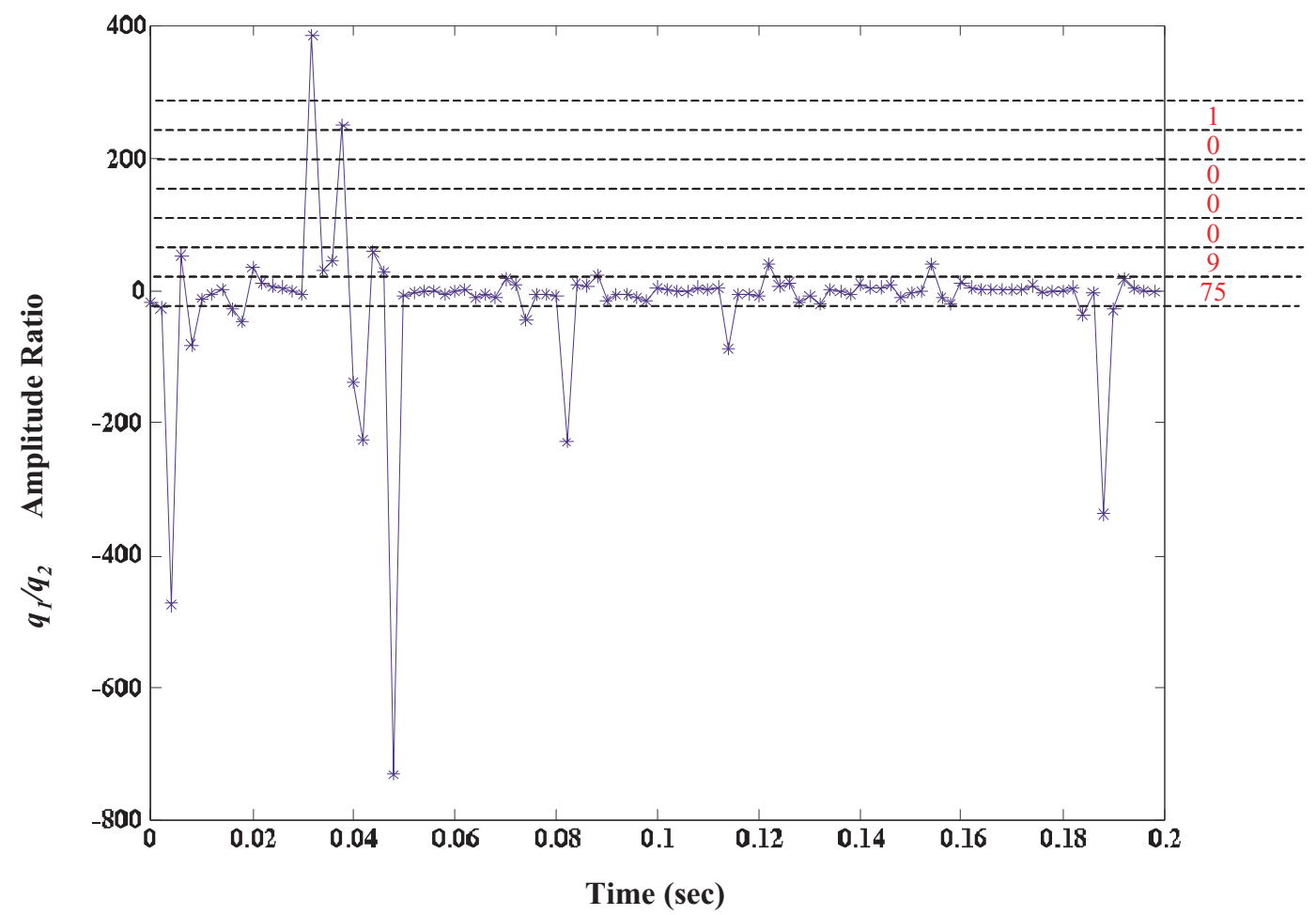

Fig. 5. Typical ratio of $q_{1} / q_{2}$ while vehicle is in motion-an example of data discretization by banding.

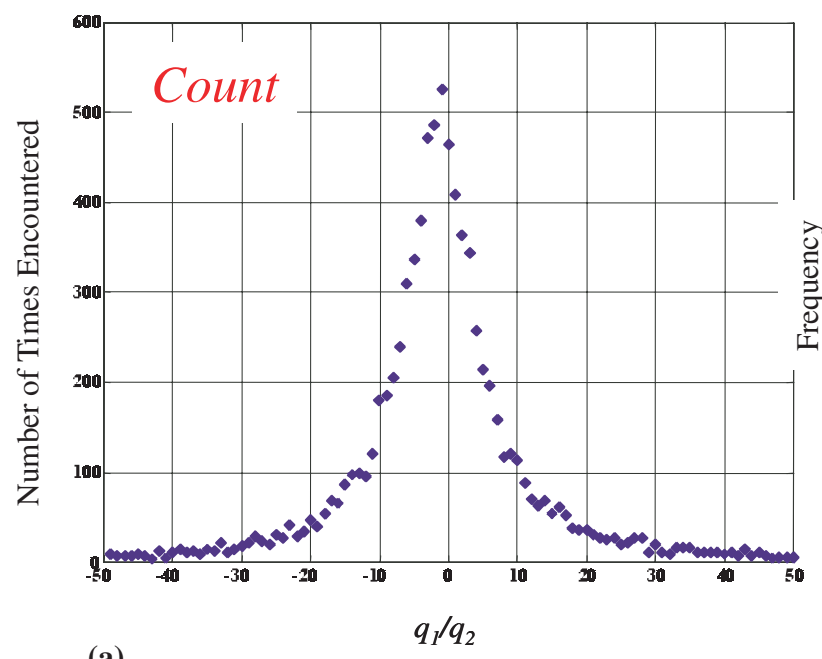

(a)

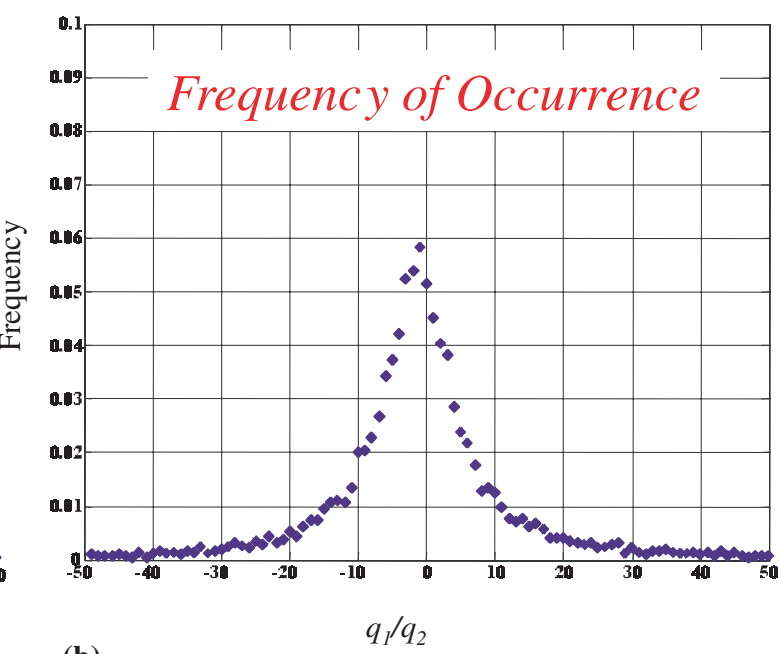

(b)

Fig. 6. Ratio of $q_{1} / q_{2}$ for an M256 barrel traversing RRC-9 bump course: (a) number of occurrences in 9,000 samples and (b) frequency of occurrence. 


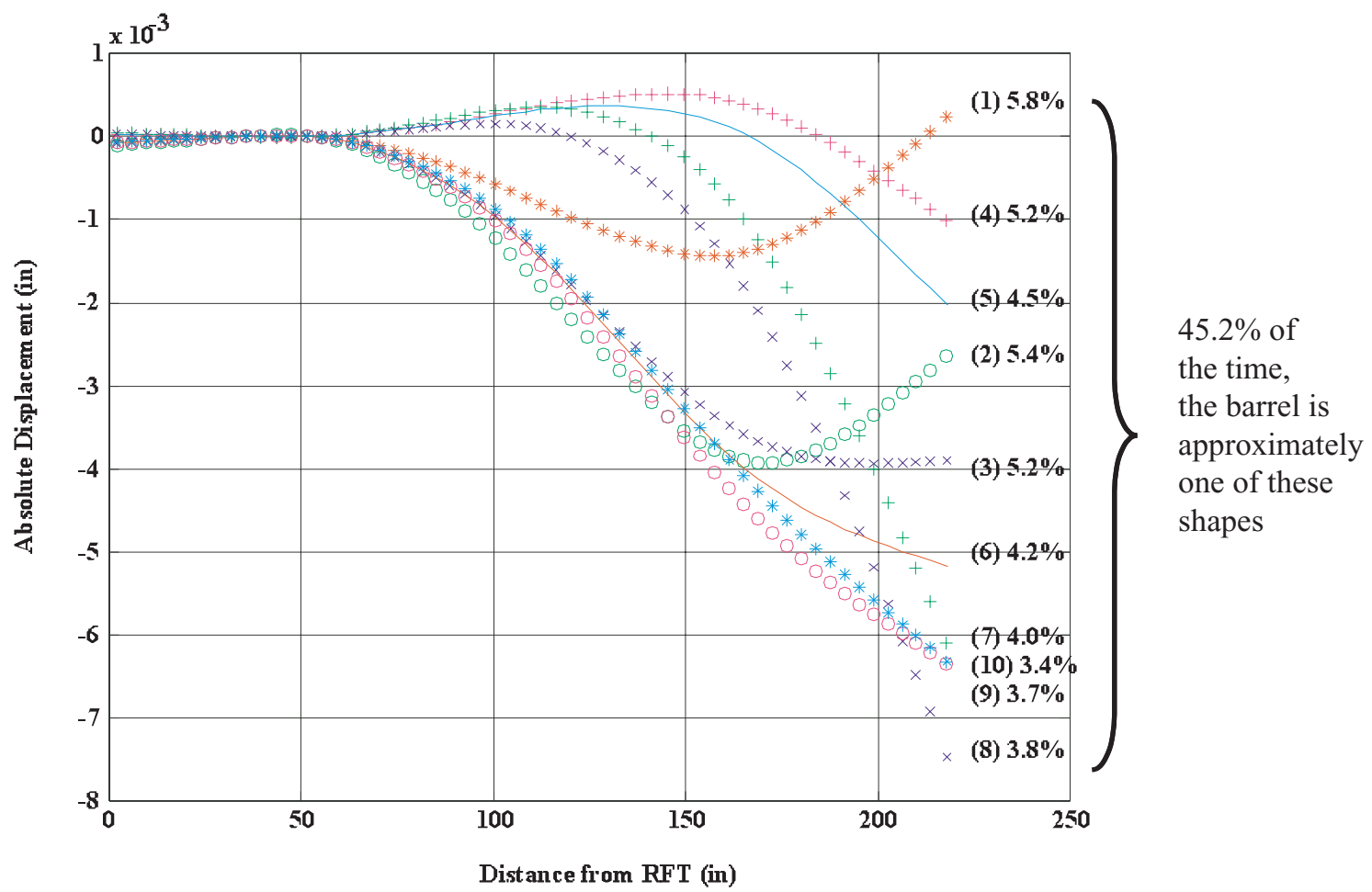

Fig. 7. Ten most likely barrel shapes over bump course.

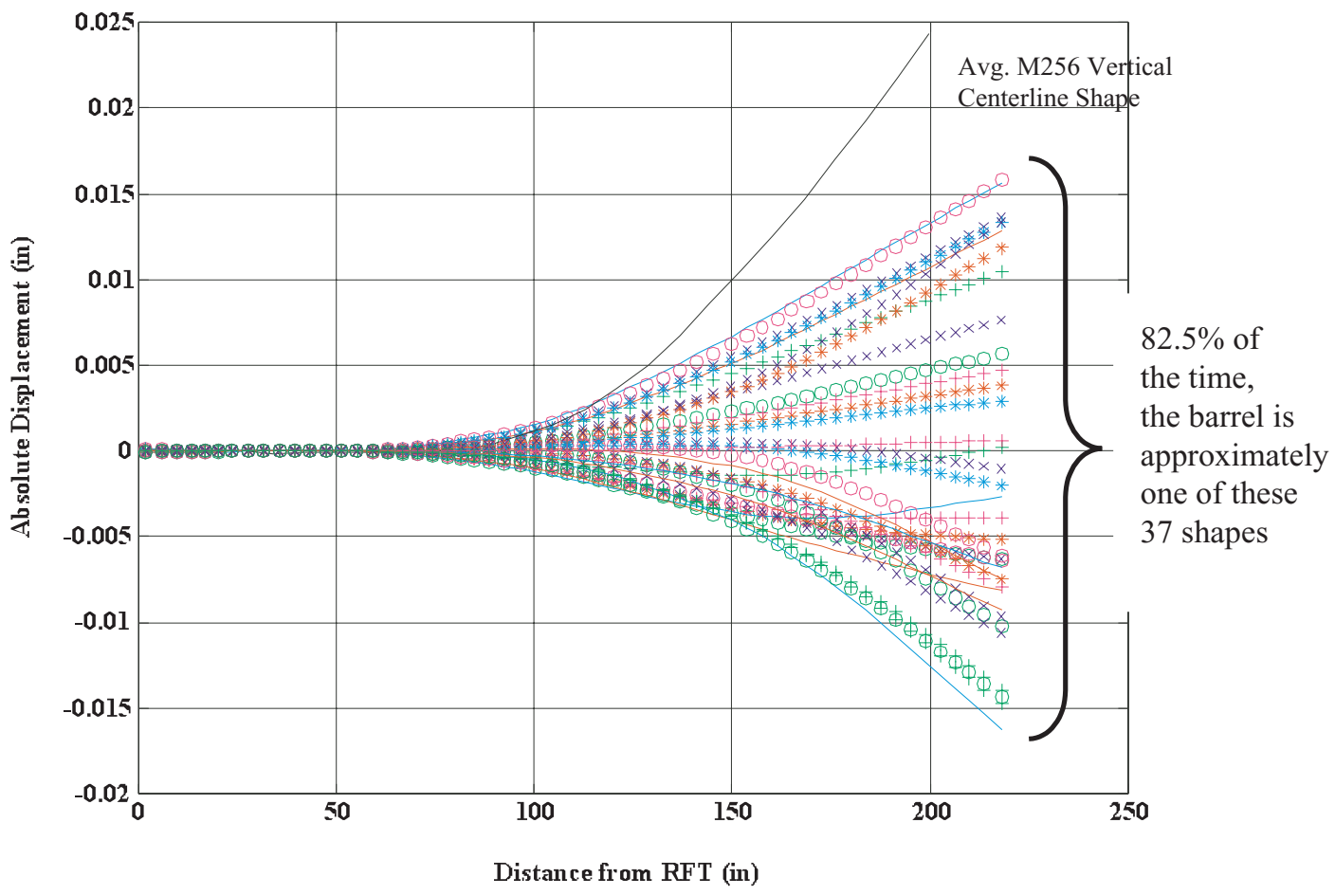

Fig. 8. Comparison of most likely bump course barrel shapes with the average manufactured shape for an M256 barrel. 

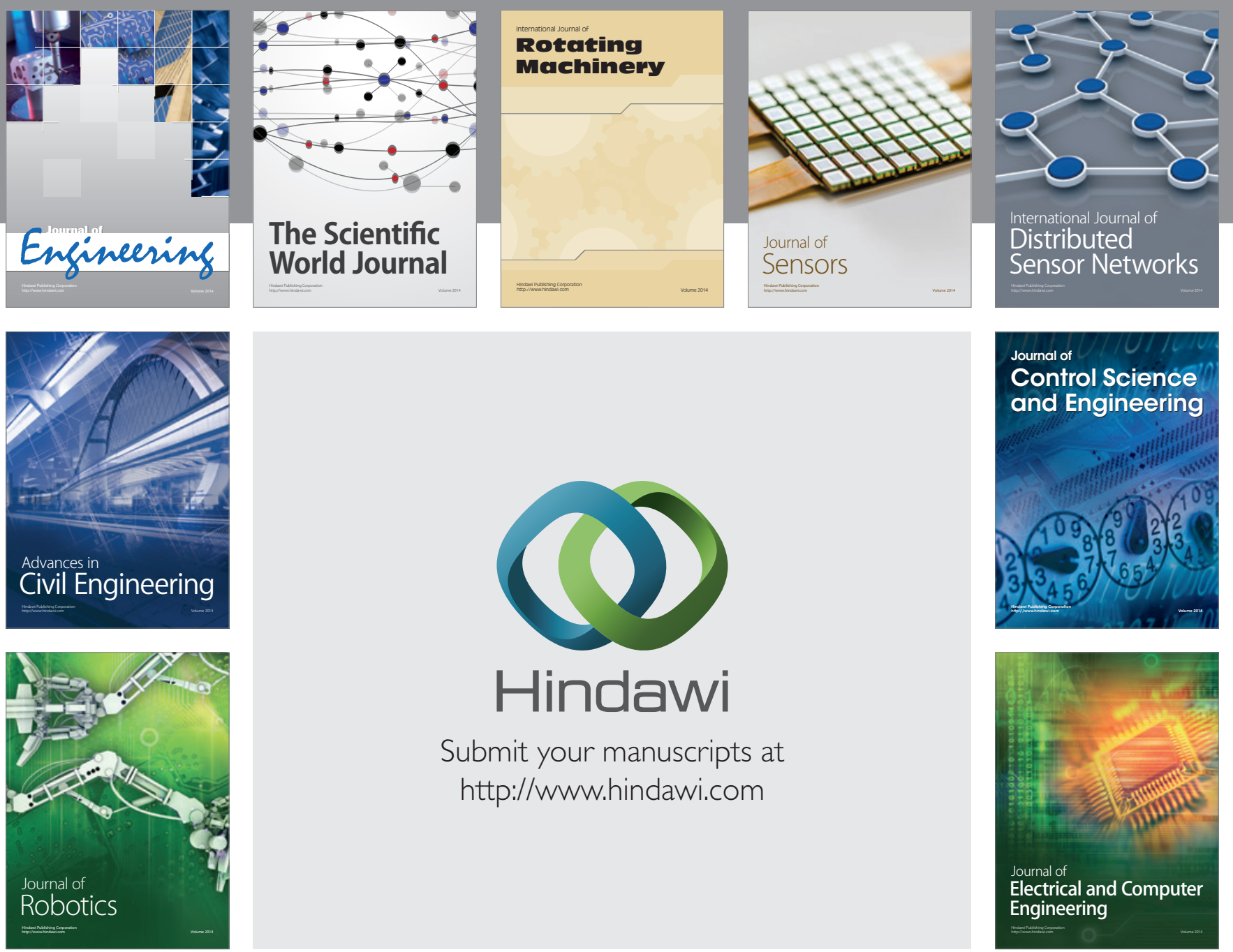

Submit your manuscripts at

http://www.hindawi.com
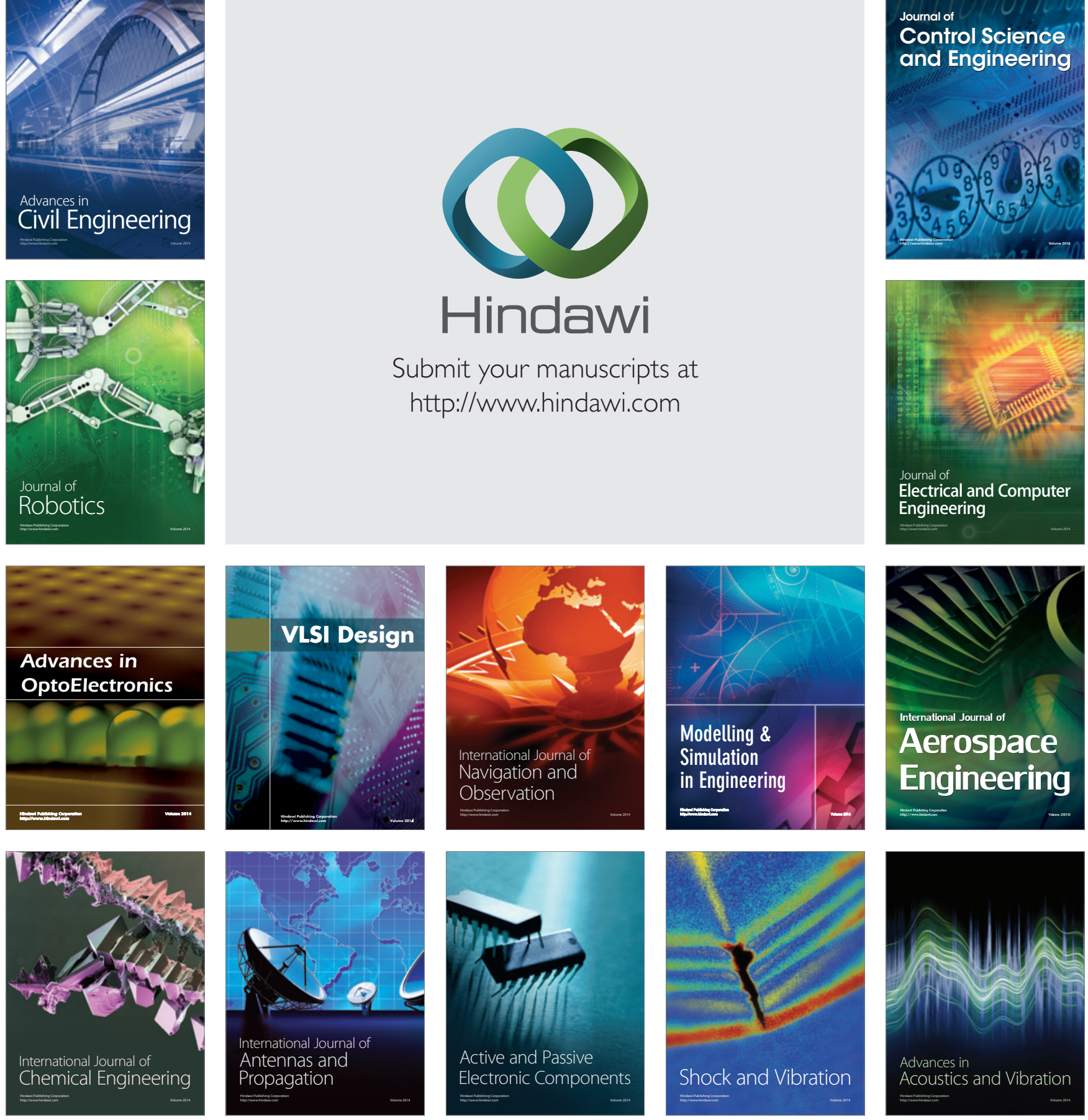